\title{
Covert Persuasion Attempts: Do People Notice? An Abstract
}

\author{
Adam D. Slobodzian and Marjorie Delbaere
}

\begin{abstract}
Pharmaceutical marketing is going through a period of transition in which many modern marketing tactics are being adopted in the pursuit of consumer engagement and brand affinity (Medical Media \& Marketing, 2015). Right now, pharmaceutical companies are using unbranded social network communities in the promotion and education of diseases through virtual support groups. Rather than using social media as a one-way promotional tool, pharmaceutical companies are using covert tactics in order to engage and monitor their target audiences (Scott Rader et al., 2013).

Much of the earlier attention in the pharmaceutical domain online has focused on company-controlled communication such as corporate web portals and drug information sites - which are both regulated-leaving an important and growing area of research focusing on tools such as social media that play an important role in consumer to consumer interaction (Scott Rader et al., 2013; Tyrawski \& DeAndrea, 2015).

The purpose of this research is to test competing theories of whether persuasion knowledge will be activated in a covert setting, specific to the context of pharmaceutical marketing. Researchers and practitioners have argued both for and against whether consumers will recognize a persuasion attempt in a covert setting, but until now there has not been a conclusive answer.

Employing an experimental design, this study is an initial step in the debate on covert marketing's effects on persuasion knowledge, specifically in the context of health and wellness communities on social media. Similar to Wojdynski and Evans' (2016) findings that consumers did not notice native advertising, the results suggest that people do not recognize these tactics, either covert or overt, as persuasion attempts; we find no significant difference in participants' evaluations of health and wellness communities when branding is present versus when it is absent.
\end{abstract}

References Available Upon Request

\footnotetext{
A.D. Slobodzian $(\varangle) \cdot$ M. Delbaere $(\varangle)$

University of Saskatchewan, Saskatoon, Canada

e-mail: adam.slobodzian@usask.ca; delbaere@edwards.usask.ca
} 